\section{TecnoLógicas}

ISSN-p 0123-7799

ISSN-e 2256-5337

Vol. 23, No. 47, pp. 213-227

Enero-abril de 2020

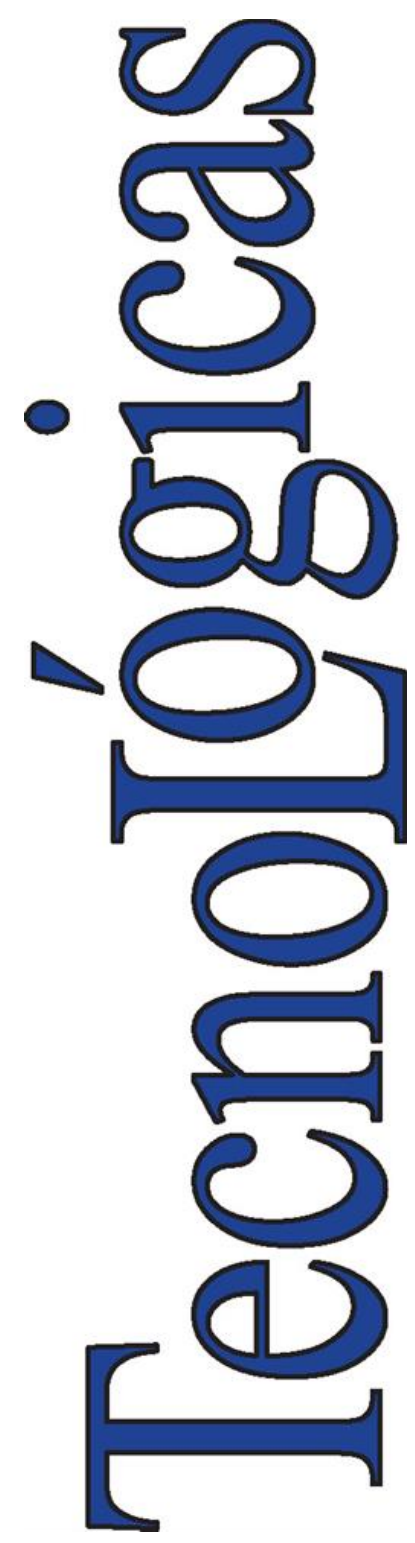

\footnotetext{
(C) Instituto Tecnológico Metropolitano Este trabajo está licenciado bajo una Licencia Internacional Creative Commons Atribución (CC BY-NC-SA)
}

Artículo de investigación/Research Article

\title{
Arquitectura de servicios adaptativos para un proceso de co- creación con estudiantes con altas capacidades
}

\section{Adaptive Services Architecture for a Co-Creation Process with High Ability Students}

\author{
Mery Yolima Uribe-Ríos (D) ${ }^{1}$, \\ Ramón Fabregat id, \\ Teodor Jové (D) ${ }_{3}$ y Montserrat Tesouro (D) 4
}

Recibido: 13 de septiembre de 2019

Aceptado: 05 de diciembre de 2019

Cómo citar / How to cite

M. Y. Uribe-Rios, R. Fabregat, T. Jové, M. Tesouro, "Arquitectura de servicios adaptativos para un proceso de co-creación con estudiantes con altas capacidades", TecnoLógicas, vol. 23, no. 47, pp. 213-227, 2020. https://doi.org/10.22430/22565337.1492

1 MSc. Ingeniería de Sistemas y Computación, Instituto de Informática y aplicaciones (IIiA), Universidad de Girona (UdG), Girona-España, mery.uribe@udg.edu

$2 \mathrm{PhD}$. en Ingeniería Informática, Instituto de Informática y Aplicaciones (IIiA). Universidad de Girona (UdG), Girona-España, ramon.fabregat@udg.edu

3 PhD. en Ingeniería Informática, Institut Instituto de Informática (IIiA), Universidad de Girona (UdG), Girona, España, teodor.jove@udg.edu

4 PhD. en Psicología, Instituto de Recerca Educativa, Universidad de Girona (UdG), Girona -España, montse.tesouro@udg.edu 


\section{Resumen}

Con el objetivo de ayudar a motivar a los estudiantes con altas capacidades en su proceso de aprendizaje, se propone que participen en la co-creación de su material educativo. Pero la participación no es suficiente, es importante hacer que esta se convierta en una experiencia personal y cercana a sus características y a su contexto. Para conseguirlo, se ha definido un proceso de co-creación de material educativo, denominado Co-CreHAs, en el que se orienta su adaptación de acuerdo a las necesidades del estudiante con altas capacidades. Esta adaptación se desarrolla en AdaptHAs, que es el módulo de adaptación de Co-CreHAs. En este artículo, se presentan el diseño y la validación de la arquitectura de los servicios adaptativos de AdaptHAs, de los cuales, el diseño se basa en el análisis de las características de las altas capacidades y del proceso de co-creación. Gracias a dicho análisis, se identificaron el tipo de perfiles de adaptación a definir, los aspectos de los participantes que se trabajarían en cada perfil y los momentos del proceso de co-creación en que se podrían usar los perfiles definidos para personalizarlo. Asimismo, la validación de la arquitectura se hace a través del análisis de tres experiencias de co-creación con estudiantes con altas capacidades, sus padres y sus profesores, para el cual se ha usado la extensión CoCreHAs para Moodle, desarrollada en el marco de la arquitectura. Con base en dicha validación, se observó que la arquitectura propuesta es una guía para la adaptación de la cocreación y para el desarrollo de un software que permita llevarla a la práctica con los servicios adaptativos definidos. También se estima que el proceso propuesto y la adaptación implementada influyen en la motivación de los estudiantes con altas capacidades frente a la co-creación de su material educativo.

\section{Palabras clave}

Co-creación de material educativo, estudiantes con altas capacidades, perfiles de adaptación, arquitectura de servicios.

\section{Abstract}

With the aim of motivating High Ability students in their learning process, we propose their participation in the co-creation of their own learning material. However, their participation is not enough because the process should also be a personal experience adapted to students' characteristics and context. For that purpose, we defined a learning material co-creation process, called Co-CreHAs, which guides the adaptation of co-created material according to High Ability students' learning needs. For said adaptation, we implemented AdaptHAs (i.e., the model used to adapt Co-CreHAs). In this paper, we present the design and validation of the architecture of adaptive services of AdaptHAs. Such architecture design is based on the analysis of the characteristics of high abilities and the co-creation process. This analysis allowed us to identify the kind of adaptation profiles that needed to be defined, participants' aspects that would be worked on in each profile, and the parts of the process where we could use those profiles in order to personalize it. The architecture was validated through the analysis of three real co-creation experiences with High Ability students, their parents, and their teachers using the Co-CreHAs extension for Moodle, which was developed applying said architecture. The results of this validation show that the architecture guides the adaptation of the co-creation and the development of software that can be implemented to put into practice such co-creation with the adaptive services previously defined. Furthermore, we observed that the proposed process and the implemented adaptation can influence High Ability students' motivation to co-create their own learning material.

\section{Keywords}

Learning material co-creation, high ability students, adaptation profiles, service architecture. 


\section{INTRODUCCIÓN}

Uno de los aspectos más importantes en todo proceso educativo es la motivación de los estudiantes. Cuando hablamos de estudiantes con altas capacidades, esta motivación puede desatarse gracias a ciertos eventos internos en la persona como pensamiento conflictivo o incertidumbre, atribuciones causales (explicaciones sobre éxito o fracaso de una tarea), emociones, sorpresa, expectativas de éxito en el futuro o el recuerdo de lo que se ha hecho [1].

La motivación aparece también por la curiosidad, como camino hacia la reducción de la incertidumbre. Según la ley "YerkesDodson", para producir curiosidad, no serían productivos ni un estado de activación de la motivación muy alto ni muy bajo [2].

Para fomentar, aumentar o inculcar la motivación a los estudiantes con altas capacidades, se decidió hacerles partícipes en la creación de su propio material educativo a través del desarrollo de procesos de co-creación. El objetivo es incentivar al estudiante en la creación de su material educativo, en un tema específico, en el trabajo grupal y en la interacción con iguales y otras personas, aspectos que se deben trabajar con los estudiantes con altas capacidades.

En relación con la co-creación, en [3] se menciona que, en la mayoría de los casos, la motivación de quienes participan en este tipo de procesos de creación es intrínseca, ya que la motivación más importante es la adquisición de conocimientos. Los participantes se motivarán por los beneficios del producto resultante, los resultados recíprocos, los sentimientos de altruismo, el aprendizaje, el apoyo a otras personas, la participación en actividades intelectualmente estimulantes, la posibilidad de expresarse y contribuir creativamente, entre otros. En [4], se menciona que el estudiante se motiva, cuando es partícipe de la creación de las actividades y tareas, en cuyo proceso se incorpora un poco de competencia en el trabajo a realizar y se otorga importancia a lo aportado por cada uno.

Así mismo, en [5] se define que "la motivación se refiere ampliamente a lo que la gente desea, lo que elige hacer y lo que se compromete a hacer". El autor explica que la motivación de un estudiante frente a su aprendizaje puede fomentarse desde el diseño de la educación dada. De esta manera, quien diseña y crea la experiencia de aprendizaje, es responsable de la motivación de quienes participan en dicha experiencia.

Por esta razón, se decidió definir un proceso de co-creación de material educativo, denominado Co-CreHAs, con base en las características de los estudiantes con altas capacidades, de manera que la experiencia fuera adaptada a sus intereses y necesidades. Esta adaptación se hace en AdaptHAs, que es el módulo de adaptación de Co-CreHAs.

En este artículo, se presentan el diseño y la validación de la arquitectura de los servicios adaptativos de AdaptHAs.

En concreto, se explica cómo se han analizado el proceso de co-creación y las características de los estudiantes con altas capacidades, para definir la arquitectura de los servicios adaptativos de AdaptHAs a considerar.

En la Sección 2, se explica el concepto de altas capacidades y el proceso de co-creación, Co-CreHAs, al que se le busca añadir características adaptativas. En la Sección 3, se describen aspectos relacionados con la adaptación tanto de la co-creación como de los estudiantes que se han considerado para definir AdaptHAs en Co-CreHAs. En la Sección 4, se presenta la arquitectura de servicios adaptativos de AdaptHAs para el proceso de co-creación.

Posteriormente, en la Sección 5, se expone la validación hecha a través del uso de la metodología Investigación Basada en el Diseño (DBR, por su nombre en inglés), para la arquitectura propuesta. 
Finalmente, en la Sección 6 se plantean las conclusiones y los trabajos futuros.

\section{CONTEXTO}

\subsection{Altas capacidades}

Las personas con altas capacidades presentan un nivel de rendimiento intelectual superior en una amplia gama de capacidades y aprenden con facilidad algún área o materia. Estas personas tienen un funcionamiento diferente a la hora de enfrentarse a una tarea $y$ resolverla [6]. Un término relacionado con las altas capacidades es el de superdotación o dotación (giftedness), el cual designa la posesión y el uso de capacidades naturales y de aptitudes sin entrenamiento y espontáneamente expresadas, en al menos un dominio, de tal manera que colocan al individuo en el $10 \%$ superior, en relación con los otros estudiantes de su edad [7].

En torno a la educación, el término altas capacidades hace parte de un grupo general de necesidades especiales de un estudiante. Este grupo se denomina ACNEAE, sigla que corresponde al Alumno Con Necesidad Específica de Apoyo Educativo. La Lomce (Ley Orgánica 8/2013, de 9 de diciembre, para la mejora de la calidad educativa) [8] define este alumnado como "aquel que requiere una atención educativa diferente a la ordinaria, por presentar necesidades educativas especiales, por dificultades específicas de aprendizaje, por tener trastorno por déficit de atención e hiperactividad (TDAH), por sus altas capacidades intelectuales, por haberse incorporado tarde al sistema educativo, o por condiciones personales o historia escolar". De acuerdo con esto, los estudiantes con altas capacidades requieren que su educación sea guiada de una manera diferente, de acuerdo con sus necesidades y características.
Dado que el aprendizaje significativo depende de la pasión y motivación de cada estudiante frente a un tema [9]-[12], con el objetivo de fomentar, aumentar o inculcar la motivación de los estudiantes en su propia educación, la propuesta es invitarlos a la co-creación de contenidos educativos para potenciar sus habilidades [13]. Con este objetivo, se utiliza la tecnología para desarrollar algunas de las habilidades personales propias de los estudiantes con altas capacidades.

Debido a que estos estudiantes tienen características especiales —que no son únicamente académicas-, que afectan su proceso de aprendizaje, se tomó la decisión de incluir en la co-creación del material académico otros dos actores: el profesor y los padres.

Generalmente, el profesor es quien desarrolla los elementos a trabajar en el proceso de enseñanza-aprendizaje, tiene el dominio de los contenidos, interactúa directamente con el estudiante e influye mayormente en su motivación para llevar a cabo una tarea [14]. Los autores de [15] describen que, generalmente, en la creación de material educativo no suele haber relación profesor-estudiante, aun cuando, algunas veces, es el estudiante quien más conoce el contenido. Por este motivo, es mucho más importante que el profesor "sepa orientar el trabajo del estudiante, que le facilite la adquisición de estrategias de búsqueda de información, que le suscite interrogantes y que equilibre sus relaciones sociales y emocionales".

Por otro lado, como se menciona en [16], en la co-creación, "los profesores tendrían un mejor resultado si trabajaran colaborativamente con los padres de familia”. En razón a esto, el segundo actor a añadir en la co-creación es la familia, pues son ellos quienes, generalmente, conocen mejor el comportamiento del estudiante a nivel académico y personal.

La decisión de incluir a la familia se basa también en varios de los modelos de definición de la dotación como el Modelo 
Global de la Superdotación [2], el modelo de Tannenbaum [17] y el modelo de Mönsk [18], que mencionan la importancia de los entornos sociales y culturales -familia, escuela, grupo de compañeros, sociedad-, en el desarrollo de sus capacidades. A fin de soportar esta idea, en [19] se describe cómo la familia debe tener una actitud activa y participativa, más allá de aportar información puntual sobre sus hijos con altas capacidades. La familia debe ser parte de la planificación de sus actuaciones en el medio familiar, social y escolar, ya que estas ayudan a que el estudiante con altas capacidades fomente y fortalezca su autoconcepto, su autonomía y su creatividad [20].

\subsection{Co-CreHAs}

La inclusión de los estudiantes con altas capacidades en la co-creación de su material educativo se hizo a través del proceso de co-creación diseñado específicamente con estudiantes con altas capacidades, que ha sido denominado Co-CreHAs (Co-creation for High Ability students) [21]. Co-CreHAs consta de seis fases: Pre co-creación, Planificar, Motivar, Desarrollar, Evaluar y Actualizar. A su vez, la fase Desarrollar se compone de cuatro subfases: Idear, Actualizar, Seleccionar y Crear (Fig. 1). Aunque las fases son secuenciales, las subfases (Idear, Analizar, Seleccionar y Crear) no lo son, necesariamente. Esto depende del desarrollo y de la dinámica del proceso de co-creación.

En las fases de Co-CreHAs hay fases "no activas" y "activas" (de color gris y verde respectivamente, en la Fig. 1). Las fases "no activas" están pensadas para antes de iniciar el proceso organizar los datos y el contexto de la co-creación y para una vez acabado actualizar los datos de acuerdo con los resultados obtenidos. Se han denominado "no activas", porque no requieren de la participación del grupo de personas que co-crean. Las fases "activas" son aquellas en las que se co-crean realmente los contenidos; en estas, el trabajo de los participantes debe ser activo todo el tiempo.

Co-CreHAs define que, para el desarrollo de las fases "activas", se debe seguir una actividad que ayude a guiar a los participantes a lograr el objetivo de la fase. Esta actividad debe ser definida al inicio de cada fase y desarrollada por todos los participantes en el proceso de co-creación. De manera general, todos los que co-crean deben participar activamente en el proceso.

Por otra parte, Co-CreHAs define tres roles para los participantes:

-Facilitador: lidera el grupo y la tarea de co-crear.

-Consultor/experto: comparte, discute y evalúa perspectivas sobre el tema a trabajar y sobre el objetivo a alcanzar.

Actúa como puente entre el contexto real y los participantes del proceso.

-Generador: colabora de forma significativa en indagar, investigar, idear, crear, prototipar y evaluar.

Para complementar los roles, Co-CreHAs define, además, un conjunto de pequeñas actividades que han sido denominadas estrategias de rol y deben ser expuestas a los participantes, para guiarlos en la representación de cada rol, de acuerdo con el objetivo de cada fase.

Estas estrategias de rol se muestran en forma de "consejo" a cada participante, según su rol y permiten que ellos tengan una idea inicial de cómo representarlo en cada parte del proceso.

Aunque la co-creación es un proceso colaborativo, no se toma como una actividad colaborativa en sí, sino como un conjunto de actividades colaborativas. De esta manera, se siguen cumpliendo las características de las actividades colaborativas como la comunicación, el feedback, el espacio de preguntas y cuestionamientos, la estructura, las tareas, los roles, la composición del grupo, entre otras [22]. 


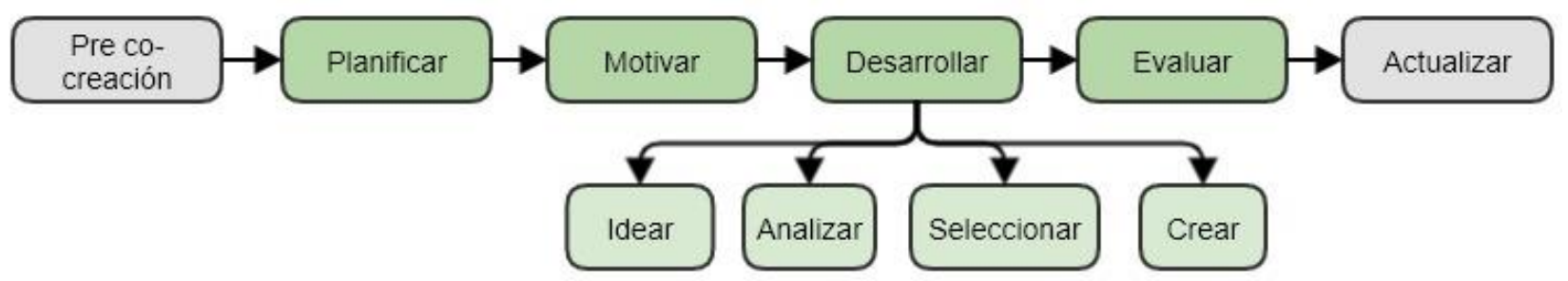

Fig. 1. Co-CreHAs. Fuente: elaboración propia.

\section{ADAPTACIÓN DE LA CO-CREACIÓN PARA LAS ALTAS CAPACIDADES: AdaptHAs}

Una vez descritas las altas capacidades y el proceso de co-creación Co-CreHAs, se analizaron ambos campos desde el punto de vista de la adaptación a la información, con el objetivo de identificar qué aspectos pueden apoyar el proceso de adaptación para los estudiantes con altas capacidades en la co-creación de contenidos educativos.

En la literatura no se encuentran trabajos relacionados con procesos colaborativos de co-creación de contenidos con estudiantes con altas capacidades. Por esta razón, el planteamiento de la adaptación se basa en trabajos que tratan las altas capacidades, la co-creación y la educación de forma independiente.

En lo referente a altas capacidades, aunque en [23] no se habla directamente de adaptación a la información, sí es quizás el estudio que más trabaja la adaptación de la información para estudiantes con altas capacidades. En este documento, se pueden resaltar aspectos de procesos adaptativos en la plataforma para el aprendizaje Renzulli, para estudiantes con altas capacidades. Entre estos aspectos, se encuentra el manejo del perfil de aprendizaje electrónico de cada estudiante, el cual opera las fortalezas, los intereses, las preferencias de estilos de aprendizajes y de modo de expresión.

Así mismo, en [24] especifican qué elementos se deben modelar del estudiante para que puedan ser aprovechados $\mathrm{y}$ adaptados a su proceso de enseñanza: conocimiento, intereses, objetivos y tareas, experiencias anteriores, contexto de trabajo y rasgos individuales (personalidad, estilos de aprendizaje, entre otros).

En relación con las altas capacidades y la co-creación, cuando esta última es gestionada por sistemas informáticos, es importante que el sistema no tome el control de los procesos [25]. Este control ayuda a remover el espacio de creatividad, opinión e interacción natural, aspectos que sobresalen en los estudiantes con altas capacidades. Con base en esta afirmación, [26] sugiere trabajar aspectos como la personalización, la conexión, la comunicación con el sistema y con los demás participantes, la interacción en tiempo real, la búsqueda y la selección de información e intercambio de roles.

A partir de estos aportes relacionados con la adaptación en las tres áreas trabajadas, se propuso que dicha adaptación se desarrollara en AdaptHAs, que es el módulo de adaptación de Co-CreHAs.

En primer lugar, se decidió trabajar la adaptación a través de un modelo de perfiles; además, en relación con las altas capacidades, se determinó incluir los aspectos más relevantes de los participantes en la co-creación en estos perfiles.

Por ejemplo, para definir el Perfil del Estudiante, se identificaron los principales aspectos a tener en cuenta acerca de estos estudiantes, entre los cuales, están:

- Características de las personas con altas capacidades: listado de 34 rasgos del comportamiento de estas personas que fueron definidos por varios autores como 
Arquitectura de servicios adaptativos para un proceso de co-creación con estudiantes con altas capacidades

[6], [27]-[32]. A continuación, se mencionan algunos de ellos:

-Tiene mucho interés en las conexiones entre los conceptos.

-Es muy sensible.

-Tiene pensamiento independiente.

-Tiene madurez para el juicio.

-Presenta liderazgo.

-Tiene habilidad para hablar.

-Manifiesta expresión no verbal.

-Tiene memoria excelente.

-Tiene pensamiento divergente.

-Tiene una gran capacidad de concentración e ignora su entorno cuando está ocupado en sus tareas, entre otras.

-Personalidad

—Estilos cognitivos

-Inteligencias múltiples

-Intereses:

-Áreas de conocimiento

-Pasatiempos

-Preferencias:

-Modos de expresión

-Estilos de aprendizaje

-Interacción

-Datos demográficos y educativos:

-Conocimientos

-Habilidades

Asimismo, se decidió crear un perfil para cada tipo de participante en el proceso de co-creación (Familia, Profesor y Social) y el perfil Contexto de co-creación que representara el entorno de los participantes durante el desarrollo del proceso.

En cuanto a Co-CreHAs, en AdaptHAs se identificó cómo podría ser adaptado el proceso de co-creación, a fin de aprovechar los aspectos de los estudiantes con altas capacidades mencionados anteriormente (ver Fig. 2).

En primer lugar, se definió que es necesario contar con la información de los participantes, para poder tener listos los perfiles de cada uno de ellos antes de iniciar el proceso.

En segundo lugar, se definieron cuatro momentos de la co-creación, para hacer la adaptación al proceso; estos son:

-MomAdapt1: asignación de roles a los participantes. Debe hacerse al inicio de la co-creación. Es importante que el rol a representar en el proceso esté de acuerdo con las características de cada participante, especialmente, en el caso de los estudiantes.

-MomAdapt2: selección del tema de la co-creación. Debe hacerse en la fase Planificación. Ayudar a guiar la selección del tema, de acuerdo a las características de los estudiantes con altas capacidades, puede aumentar su motivación en el proceso.

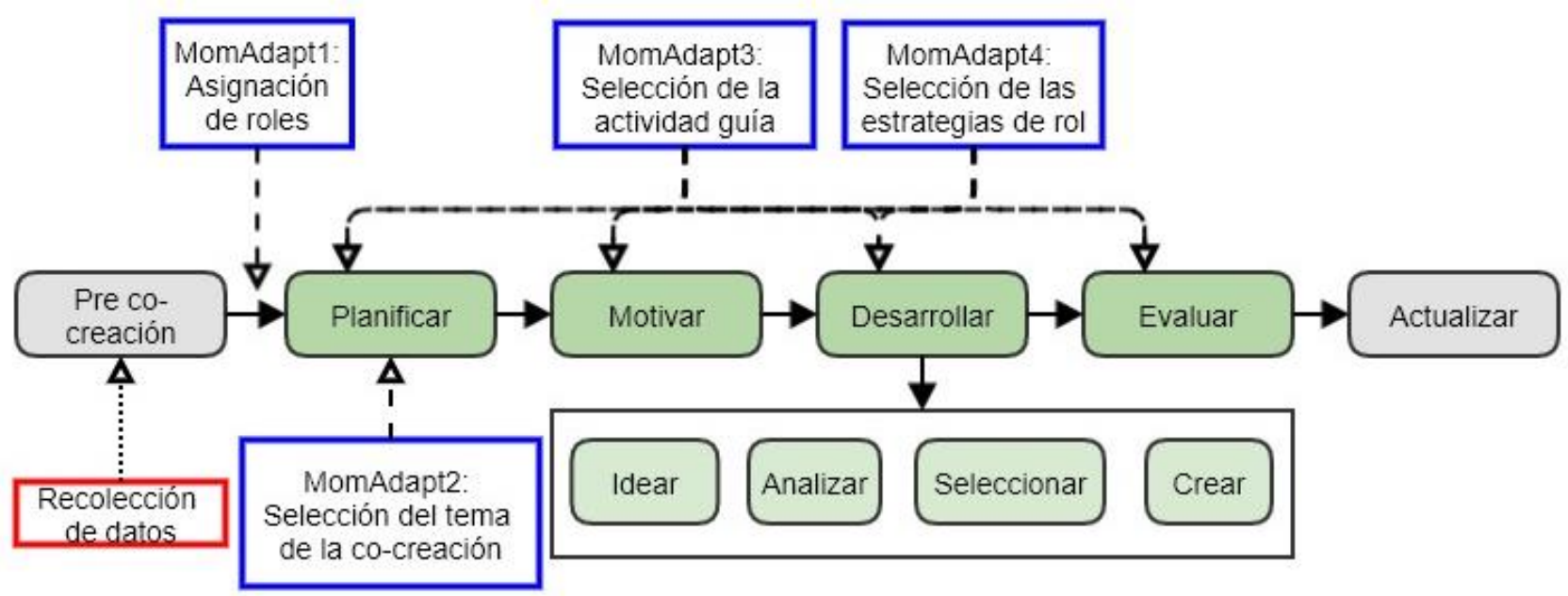

Fig. 2. Adaptación para Co-CreHAs. Fuente: elaboración propia. 
-MomAdapt3: selección de la actividad guía de cada una de las fases, la cual se debe hacer al inicio de estas. El objetivo es que esta actividad esté de acuerdo con las características de los participantes, de manera que sea fácil de entender y los motive en el desarrollo de esa fase.

-MomAdapt4: selección de las estrategias de rol para cada participante.

Debe hacerse al inicio de cada una de las fases del proceso. Las estrategias de rol son importantes no solo para apoyar a nivel individual a los participantes en su rol, sino también para ayudar a equilibrar el trabajo en grupo, a través de los roles de cada uno. Por esta razón, la adaptación se hace con base en los aspectos individuales y del contexto de la co-creación.

\section{ARQUITECTURA DE SERVICIOS ADAPTATIVOS PARA Co-CreHAs}

Una vez definidos los cuatro momentos en los que se haría la adaptación en Co-CreHAs, se pasó a diseñar la arquitectura de AdaptHAs, para poder hacer la adaptación (ver Fig. 3). Tal como se menciona en [33], esta arquitectura se definió para desacoplar el desarrollo de la lógica de adaptación a la información de la lógica del desarrollo del proceso de co-creación Co-CreHAs.

El objetivo era cubrir las necesidades de adaptación propias del proceso de co-creación Co-CreHAs. Con este fin, se diseñó una arquitectura simple, pero que diera respuesta a lo identificado para el proceso de co-creación en cuanto a la adaptación. Por esta razón, no se optó por diseños genéricos de arquitecturas como las mencionadas en [34], sino por un diseño en capas desacopladas, con un enfoque progresivo y abierto, que permitiera una innovación colaborativa como se propone en [35].
En la primera capa (Modelo de datos), se encuentran los datos que son la base de la adaptación, en este caso, el Modelo de Adaptación (compuesto por los perfiles Estudiante, Familia, Profesor, Social y Contexto de co-creación) y el Modelo del Dominio (compuesto por los datos de Actividad, Proceso de co-creación y Material educativo). Para la recolección de los datos de los perfiles de los participantes se diseñó un conjunto de tests.

En la segunda capa (Gestión de Cocreación), están los paquetes a desarrollar para gestionar los cuatro aspectos principales en el proceso de co-creación: interacción, aportes, consulta de información y el proceso de co-creación como tal.

En la tercera capa (Servicios adaptados para Altas Capacidades), se hallan los servicios adaptativos para Co-CreHAs, de acuerdo con los momentos de adaptación definidos anteriormente. En [36], se ofrece una explicación más detallada de los tres primeros servicios de AdaptHAs.

Debido a que esta es una arquitectura por capas, cada una de estas depende de las capas anteriores. Así, para gestionar el proceso de co-creación son necesarios los datos del Modelo de Adaptación y del Modelo del Dominio. Una vez el proceso y sus aspectos son gestionados, pueden llevarse a cabo los servicios adaptativos, es decir, se puede adaptar Co-CreHAs en los cuatro momentos definidos.

Por último, la cuarta capa (Aplicación) se designa para la aplicación de software que manejará Co-CreHAs. Esta capa se desarrolló a través de una extensión para Moodle llamada Co-CreHAs, creada para guiar el proceso de co-creación con la implementación de los cuatro servicios adaptativos. 
Arquitectura de servicios adaptativos para un proceso de co-creación con estudiantes con altas capacidades

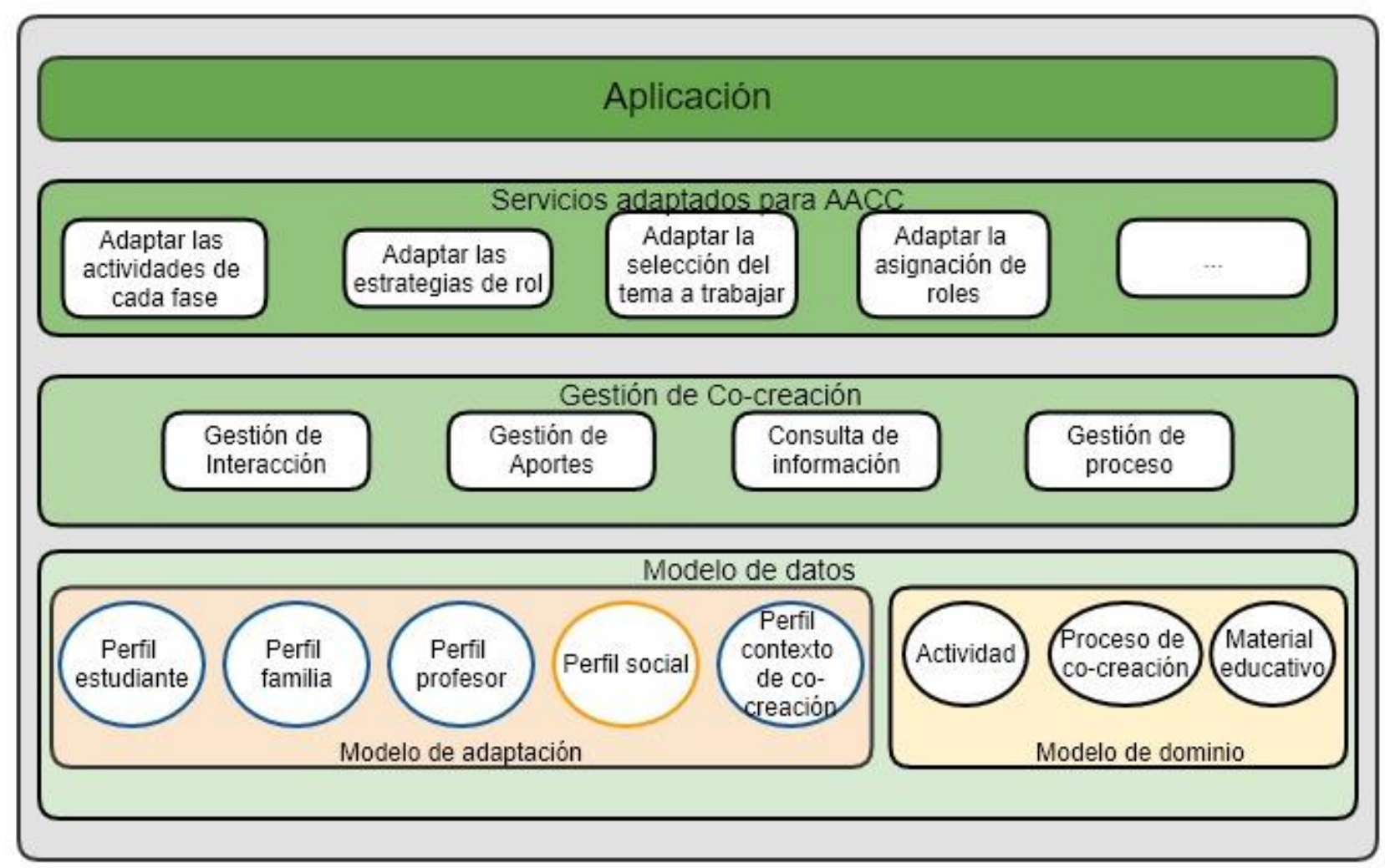

Fig. 3. Arquitectura de servicios adaptativos para Co-CreHAs

Fuente: elaboración propia.

\section{VALIDACIÓN DE LA ARQUITECTURA}

Una vez diseñada la arquitectura, el siguiente paso fue validarla a través del uso de la extensión Co-CreHAs para Moodle. El objetivo era verificar que la arquitectura cumpliera con el diseño dado (orden, uso y comunicación entre capas) y que diera soporte a la adaptación de la información definida para el proceso de co-creación. Esta validación se trabajó bajo la metodología Investigación Basada en el Diseño (DBR, por su nombre en inglés) [37], [38]. La metodología DBR es "una metodología sistemática pero flexible dirigida a mejorar las prácticas educativas a través del análisis iterativo, el diseño, el desarrollo y la implementación basados en la colaboración entre investigadores y profesionales en entornos del mundo real y conduciendo a teorías y principios de diseño sensibles con el contexto" [38].
La metodología DBR nos permitió observar el comportamiento de la extensión Co-CreHAs, desde el punto de vista del usuario final y mejorar las sucesivas experiencias, de acuerdo con los resultados obtenidos en cada una.

Para validar cada capa de la arquitectura se definieron las acciones que se debían seguir. Para la capa "Modelo de datos", se solicitaron los datos de los participantes en la fase de "pre co-creación" de Co-CreHAs y así completar el Modelo de Adaptación. La capa "Gestión de la co-creación" fue cubierta desde el desarrollo de la extensión para Moodle, de manera que la gestión quedara implícita en la programación del software. En la capa "Servicios adaptados para Altas Capacidades", se implementaron los algoritmos que manejan la adaptación en tiempo de ejecución de la co-creación y se hizo hincapié en la necesidad de dichos servicios para los participantes a la hora de 
desarrollar la co-creación. Para la capa "Aplicación", se desarrolló la extensión Co-CreHAs para Moodle, que gestiona el proceso de la co-creación.

Una vez definido lo necesario para trabajar cada capa de la arquitectura, se establecieron los parámetros de la adaptación que se deberían manejar en la validación. De acuerdo con [39], en la evaluación de sistemas adaptativos se debe asegurar que el sistema se comporte según el diseño y que cumpla los requerimientos de los usuarios. Así mismo, se deben evaluar los requerimientos y la funcionalidad e identificar posibles problemas a través del feedback de los usuarios del sistema. No se deben usar grupos de control en sistemas adaptativos, porque la adaptación para dos grupos debería ser diferente. Tampoco se debe trabajar el mismo grupo sin $\mathrm{y}$ con adaptación, porque hacerlo sin adaptación no tiene sentido. Los aspectos observados y medidos fueron:

- Cantidad de datos solicitados

- Correcta categorización de usuarios (respecto a los roles)

-Tiempo de cálculo de los métodos de adaptación

- Conocimiento del dominio; ganancia de aprendizaje

-Impresión general

- Calificación de la calidad de la solución

-Valoración subjetiva

-Éxito de la tarea

- Satisfacción del usuario

Para llevar a cabo la validación de Co-CreHAs, se utilizó la metodología DBR con un grupo de tres estudiantes con altas capacidades en un total de tres experiencias, denominadas E1, E2 y E3, respectivamente, en las cuales los participantes usaron la extensión de Co-CreHAs en Moodle. En la experiencia E1, el grupo no trabajó los roles en la co-creación, debido a que se enfocaron en el desarrollo del proceso como tal y no pusieron atención a sus roles. Por este motivo, no se validaron todos los servicios de la capa tres y se vio la necesidad de definir una nueva experiencia (la E2), en la que se trabajaron los roles. Sin embargo, como no se llegaron a usar las actividades propuestas por Co-CreHAs en cada fase, se tuvo que hacer la experiencia E3, en la que sí se usaron las actividades propuestas por Co-CreHAs.

De manera general, se pudo observar que las características de las actividades colaborativas tenidas en cuenta por el proceso de co-creación Co-CreHAs fueron soportadas también por la arquitectura propuesta. Por ejemplo, comunicación, feedback, espacio de preguntas y cuestionamientos recibieron soporte de los gestores de la capa dos. Asimismo, estructura, tareas, roles y composición de grupo se soportaron gracias a los servicios adaptados de la capa tres.

Desde su inicio, todos los participantes estuvieron muy involucrados en cada experiencia. Los participantes con más dudas a la hora de llevar a cabo el proceso fueron los padres de familia, pero siempre respondían con interés frente a las dudas, las novedades (que introducían los estudiantes) y los retos planteados durante el proceso de co-creación.

$\mathrm{Al}$ terminar cada una de las experiencias, se midieron los aspectos de adaptación definidos. Cada aspecto fue evaluado en una escala de 1 a 3 (1-Bajo, 2Medio y 3-Alto) y sus resultados se exponen en la Tabla 1:

En relación con los aspectos de adaptación, se observó que, de manera general, ningún aspecto fue calificado como Bajo y que, según el promedio de cada uno, 6 de los 9 aspectos fueron calificados como Alto.

Los participantes de las experiencias expresaron que los datos solicitados en la fase "Pre co-creación" fueron bastantes, pero que el cumplimiento de los formularios les ayudó a conocerse mejor. 
Arquitectura de servicios adaptativos para un proceso de co-creación con estudiantes con altas capacidades

Tabla 1. Resultados de la validación de la arquitectura. Fuente: elaboración propia.

\begin{tabular}{lcrrrr}
\hline \multicolumn{1}{c}{ Aspecto } & E1 & E2 & E3 & Promedio \\
\hline Cantidad de datos solicitados & 2 & 2 & 2 & 2 \\
Correcta categorización de usuarios (respecto a los roles) & 3 & 2 & 2 & $2,3 \approx 2$ \\
Tiempo de cálculo de los métodos de adaptación & 3 & 3 & 3 & 3 \\
Conocimiento del dominio; ganancia de aprendizaje & 2 & 3 & 3 & $2,7 \approx 3$ \\
Impresión general & 2 & 3 & 3 & $2,7 \approx 3$ \\
Calificación de la calidad de la solución & 2 & 2 & 2 & 2 \\
Valoración subjetiva & 3 & 2 & 3 & $2,7 \approx 3$ \\
Éxito de la tarea & 3 & 3 & 3 & 3 \\
Satisfacción del usuario & 3 & 3 & 3 & 3 \\
\hline
\end{tabular}

Sobre los roles, los participantes dijeron que se identificaban con las características del rol asignado, pero llamó la atención que, tanto en la E2 como en la E3, los estudiantes con el rol "Consultor/Experto" mencionaron que hubieran estado más cómodos con el rol de "Generador". Sin embargo, ambos estudiantes desempeñaron muy bien su rol en cada experiencia, especialmente, el estudiante Consultor/Experto de la E3.

Respecto al "Tiempo de cálculo" utilizado por Co-CreHAs (medido directamente a nivel software) para desarrollar los diferentes métodos, este fue muy corto. El tiempo máximo entre inicio y fin de un método fue, como máximo, un segundo.

En relación con los items Impresión general, Calidad, Valoración, Éxito y Satisfacción, se puede resaltar que todos ellos tuvieron una evaluación semejante en las tres experiencias, pero los participantes estuvieron de acuerdo en que, con cada experiencia nueva, el ejercicio era mejor y se sentían más cómodos en el proceso, porque ya lo conocían y esto les permitía centrarse en otros elementos como las actividades por fase o las estrategias de rol.

A través de nuestra participación dentro del proceso de co-creación, como lo indica la metodología DBR, se estima que los participantes iban incrementando su motivación tanto por el proceso como por el tema manejado en la co-creación. A medida que cada experiencia avanzaba, los participantes se apropiaban más del proceso y de los aportes que pudieran contribuir a la co-creación.

Frente al tema de la arquitectura, el comportamiento de Co-CreHAs en cada capa fue satisfactorio.

Para la primera capa, se vio que los datos almacenados en cada perfil fueron manejados de forma personalizada para cada participante y de forma adaptativa para el grupo y sus acciones colaborativas.

La segunda capa pudo gestionar estos datos a través de la Interacción, los Aportes, la Consulta de información y el manejo del Proceso. No se presentaron inconvenientes con el acceso de los datos del Modelo de la primera capa y estos pudieron ser entregados de forma correcta a la tercera capa.

En la tercera capa, para cada uno de los servicios adaptativos y los momentos de adaptación, podemos mencionar que:

-MomAdapt1-Asignación de roles: los participantes manifestaron que se sintieron cómodos con el rol de cada uno y que se sentían identificados con su papel.

En la experiencia E3, el Consultor/Experto mencionó que se hubiera sentido más cómodo como Generador, aunque hizo muy bien su papel y obtuvo muy buena calificación por parte de los demás participantes.

-MomAdapt2-Actividad propuesta en cada fase: estas actividades fueron desarrolladas a conciencia en las fases Planificar, Motivar y Evaluar. En la fase Desarrollar, estas actividades fueron un poco 
olvidadas, especialmente en la subfase Crear, pues los participantes estaban muy involucrados en el proceso y terminaron co-creando el material, por lo que dejaron un poco de lado la actividad.

Esto se vio, principalmente, en los estudiantes más dispersos.

-MomAdapt3-Sugerencias de temas: el tema seleccionado en la experiencia E1 fue uno de los sugeridos por Co-CreHAs. En las experiencias E2 y E3, en cada grupo, uno de los estudiantes se inclinó por trabajar una de las sugerencias de tema, pero los demás no; por lo tanto, trabajaron temas del sistema, que no eran parte de las sugerencias.

-MomAdapt4-Selección de estrategias de rol: se observó que las estrategias ayudaron a nivelar y encaminar el trabajo de los grupos, especialmente en momentos en que los estudiantes se distraían. En varias ocasiones, en este contexto, se observó el uso de las estrategias en los padres de familia y en el estudiante con el rol "Facilitador" de la experiencia E3.

Finalmente, la cuarta capa tuvo un buen desempeño gracias a las tres primeras capas, pues la interacción usuario-sistema no tuvo inconvenientes de tiempos de ejecución, acceso a sus contenidos ni entendimiento por parte de los participantes.

Tras estas observaciones, se puede decir que la arquitectura propuesta:

-Da soporte a la adaptación a la información para Co-CreHAs, pues los servicios adaptativos se desempeñaron según lo planificado y adaptaron el proceso de co-creación a sus necesidades iniciales.

-Permite que se agreguen servicios adaptativos de forma fácil y manejable.

Durante el desarrollo de estas tres experiencias, se observó que la segunda capa podría mejorarse. Para este fin, una de las opciones es crear un gestor para el ingreso de los datos que se manejan en la primera capa. De esta manera, los datos se manejarían desde la misma extensión y no se necesitarían formularios y aplicaciones externas. Otra mejora consiste en desarrollar un gestor que permitiera un mejor manejo de las herramientas internas y de los datos de Moodle, para poder sacar más provecho de la plataforma en el proceso de co-creación.

Con base en todos los resultados, se puede anotar que la arquitectura funciona según el diseño propuesto. Las capas inferiores son indispensables para las capas superiores y éstas se comunican entre sí, sin ningún problema. A pesar de que los servicios necesitan los datos de los perfiles de forma constante en la ejecución de los algoritmos en cada fase y subfase, para cada participante del proceso, no se observó una limitación en su acceso o demoras de comunicación entre las capas.

\section{CONCLUSIONES}

Este artículo expone la forma en que se diseñó la arquitectura de servicios adaptativos de AdaptHAs, módulo de adaptación de Co-CreHAs, que es un proceso de co-creación de material educativo para estudiantes con altas capacidades. El diseño de dicha arquitectura se desarrolló por capas, en la que cada una es dependiente de la capa anterior. Especialmente, se presentó la manera en que fueron seleccionados los datos de la primera capa, con el objetivo de estimular el desarrollo de las características de los estudiantes con altas capacidades y aquellos servicios que fueron seleccionados e implementados para adaptar la co-creación.

Frente al diseño de la arquitectura, se concluye que el diseño por capas permite enfatizar los datos y características de los estudiantes con altas capacidades, enfocar el desarrollo de los servicios en estos datos e incluir en la co-creación aquellas personas más cercanas a estos estudiantes, quienes pueden influir en su motivación.

Además, este diseño facultó un desarrollo viable de la extensión de software Co-CreHAs para Moodle. Así, el desarrollo se centró primero en la organización de los datos de los perfiles, posteriormente, en 
gestionar el proceso y, finalmente, en la implementación de los servicios adaptativos.

El uso de la metodología DBR hizo posible enfocar las experiencias de la validación en los servicios adaptativos y en la percepción de los participantes sobre esta adaptación. De esta manera, cada experiencia pudo ser enfocada en los elementos que necesitaban más atención en cada validación.

Asimismo, con la puesta en marcha de las experiencias para la validación de la arquitectura de AdaptHAs, se pudo corroborar que el diseño permitía la comunicación de los servicios y los datos, sin representar carga para el sistema o demora en la interacción del sistema con los participantes de la co-creación.

A partir de los resultados de los aspectos de adaptación evaluados en las tres experiencias reales, se puede afirmar que la arquitectura diseñada brinda soporte a los servicios adaptativos y a las características propias de colaboración que maneja el proceso de co-creación. En consecuencia, la co-creación puede ser un proceso adaptado al entorno colaborativo de cada grupo, que trabaje las particularidades de cada uno de los participantes, especialmente de los estudiantes con altas capacidades.

Finalmente, se estima que la motivación de los estudiantes se vio fortalecida, gracias a que los servicios se adaptaron con base en los datos personales de cada participante; esto hizo que la co-creación fuera una experiencia más cercana para ellos.

\section{AGRADECIMIENTOS}

Al programa IF_UDG2015 de la UdG, al proyecto Open Co-creation (TIN2014-53082R) financiado por el Ministerio Español de Economía y Competitividad, y al grupo de investigación BCDS (GRCT40) que forma parte del grupo consolidado Smart IT Engineering and Services (SITES) (ref. 2017 SGR-1551).

\section{REFERENCIAS}

[1] M. Martínez i Torres, À. Guirado Serrat, Altas capacidades intelectuales pautas de actuación, orientación, intervención y evaluación en el periodo escolar. España: Grao, 2012. Disponible en: URL

[2] L. Pérez Sánchez y P. Domínguez Rodríguez, Superdotación y adolescencia. Características y necesidades en la comunidad de Madrid. Madrid: Consejería de Educación. Comunidad de Madrid, 2001. Disponible en: URL

[3] T. Fernandes and P. Remelhe, "How to engage customers in co-creation: customers' motivations for collaborative innovation," $J$. Strateg. Mark., vol. 24, no. 3-4, pp. 311-326. 2016.

https://doi.org/10.1080/0965254X.2015.109522 $\underline{0}$

[4] L. Ferlazzo, "Strategies for Helping Students Motivate Themselves", Edutopia, 25 mar. 2015. Disponible en: URL

[5] J. M. Keller, Motivational Design for Learning and Performance The ARCS Model Approach. Boston, MA: Springer US, 2010. https://doi.org/10.1007/978-1-4419-1250-3

[6] J. C. Torrego et al., Alumnos con altas capacidades y aprendizaje cooperativo. Un modelo de respuesta educativa. Madrid: Fundación SM, 2011. Disponible en: URL

[7] F. Gagné, "A Differentiated Model of Giftedness and Talent (DMGT)," 2000. Disponible en: URL

[8] Boletín oficial del estado, Ley Orgánica 8/2013, de 9 de diciembre, para la mejora de la calidad educativa. BOE-A-2013-12886 España, 2013, pp. 2-64.Disponible en: URL

[9] A. Bedoya Córdoba et al., "Diseño e implementación de protocolos de intervención neuropsicopedagógica fase III," Universidad de Manizales,/ Especialización en Neuropsicopedagogía) Facultad de Ciencias Sociales y Humanas 2008. Disponible en: URL

[10] A. Grant, "How to Raise a Creative Child. Step One: Back Off," New York Times, 2016. Disponible en: URL

[11] B. Oakley, O. Trejo y T. Sejnowski, Coursera, Aprendiendo a aprender: Poderosas herramientas mentales con las que podrás dominar temas difíciles, 2016. Disponible en: URL

[12] E. Punset y Corporación Radio Televisión Española, Redes. El sistema educativo es anacrónico. 2011. Disponible en: URL

[13] F. Garnett y N. Ecclesfield, "Towards a framework for co-creating Open Scholarship," Res. Learn. Technol., vol. 19, pp. 5-17, Jan. 2011. Disponible en: URL 
Arquitectura de servicios adaptativos para un proceso de co-creación con estudiantes con altas capacidades

[14] D. Siegle, L. D. Rubenstein, y M. S. Mitchell, "Honors Students' Perceptions of Their High School Experiences: The Influence of Teachers on Student Motivation," Gift. Child Q., vol. 58, no. 1, pp. 35-50, Dec. 2014.

https://doi.org/10.1177/0016986213513496

[15] C. Genovard Roselló y A. Castelló, El límite superior: aspectos psicopedagógicos de la excepcionalidad intelectual. España: Ediciones Pirámide, 1990. Disponible en: URL

[16] L. Ferlazzo, "Response: Seeing Families as 'Co-Creators' of our Schools," Education Week Classroom Q\&A With Larry Ferlazzo, 2016. Disponible en: URL

[17] A. J. Tannenbaum, "The meaning and making of giftedness", Handb. Gift. Educ., vol. 2, 27-42, 1997.

[18] F. J. Mönsk, M. W. Katzko, and H. W. Van Boxte, Education of the Gifted in Europe: Theoretical and Research Issues (Part A: Volume 28). Amsterdam: Swets \& Zeitlinger, Amsterdam, 1992. Disponible en: URL

[19] M. i Torres, "Pautas de intervención para la colaboración familia-escuela en el alumnado de altas capacidades intelectuales," in $I V$ Jornadas de respuesta educativa a las altas capacidades, Murcia, 2008. Disponible en: URL

[20] D. C. Simons, A. B. Ramírez, A. M. F. Belmont, and C. A. H. Rodríguez, "Apoyo familiar en escolares de alta capacidad intelectual de diferentes contextos socioeducativos," Rev. Psicol., vol. 33, no. 2, pp. 299-332, 2015. Disponible en: URL

[21] M. Y. Uribe-Rios, T. Jove, R. Fabregat, y J. P. Meneses-Ortegon, "Co-CreHAs: Co-creation process of enrichment activities for high ability students," en 2018 IEEE Global Engineering Education Conference (EDUCON), Tenerife, 2018. pp. 1645-1651.

https://doi.org/10.1109/EDUCON.2018.8363432

[22] B. M. Hall, "Designing Collaborative Activities to Promote Understanding and ProblemSolving," Int. J. e-Collaboration, vol. 10, no. 2, pp. 55-71, Apr. 2014.

https://doi.org/10.4018/ijec.2014040104

[23] J. S. Renzulli, S. M. Reis, B. Wallace, y G. Erikson, "A virtual learning application of the schoolwide enrichment model and high-end learning theory," Gift. Educ. Int., vol. 28, no. 1, pp. 19-40, Jan. 2012.

https://doi.org/10.1177/0261429411424382

[24] P. Brusilovsky y E. Millán, "User Models for Adaptive Hypermedia and Adaptive Educational Systems," in The Adaptive Web, P. Brusilovsky, A. Kobsa, and W. Nejdl, Eds. Berlin, Heidelberg: Springer Berlin Heidelberg, 2007. pp. 3-53. https://doi.org/10.1007/978-3-540-72079-9_1
[25] S. J. McMillan, "Exploring Models of Interactivity from Multiple Research Traditions: Users, Documents, and Systems," en Handbook of New Media: Social Shaping and Consequences of ICTs, 1 Oliver's Yard, 55 City Road London EC1Y 1SP: SAGE Publications, Ltd, 2010. pp. 163-182. https://doi.org/10.4135/9781848608245.n13

[26] D. E. Kim, "The impact of smart device's interactivity on customer activity in the sports industry," (Tesis de Maestría) Arkansas State University, United States, Arkansas, 2014. Disponible en: URL

[27] American Mensa, Ltd., Gifted characteristics, 2016. Disponible en: URL

[28] B. Kingore, "High Achiever, Gifted Learner, Creative Thinker," Underst. Our Gift., vol. 15, no. 3, 2003. Disponible en: URL

[29] National Association for Gifted Children, "Common Characteristics of Gifted Individuals,". Disponible en: URL

[30] Newfoundland and Labrador Department of Education, Teaching students who are gifted and talented a Handbook for Teachers. 2013. Disponible en: URL

[31] C. A. Tomlinson, How to Differentiate Instruction in Mixed-ability Classrooms, 2nd ed. Alexandría Virginia: Association for supervition and Curriculum Development, 2001. Disponible en: $\underline{\mathrm{URL}}$

[32] J. T. Webb, J. L. Gore, E. R. Amend, and A. R. DeVries, A Parent's Guide to Gifted Children. Arizona: Great Potential Press, Inc., 2007. Disponible en: $\underline{\mathrm{URL}}$

[33] J. Dowling and V. Cahill, "The K-Component Architecture Meta-Model for Self-Adaptive Software," in Metalevel Architectures and Separation of Crosscutting Concerns, Berlin, Heidelberg: Springer, 2001. pp. 81-88. https://doi.org/10.1007/3-540-45429-2_6

[34] J. F. Inglés Romero y C. Vicente Chicote, "Guías de diseño para sistemas adaptativos basados en componentes," in V Jornadas de introducción a la investigación de la UPCT, 2012, pp. 96-98. Disponible en: URL

[35] D. Martín, "Diseño de aplicaciones adaptativas," No Solo Usabilidad, no. 9, 2010. Disponible en: URL

[36] M. Y. Uribe-Rios, T. Jové, R. Fabregat, y J. P. Meneses-Ortegón, “AdaptHAs: Adapting Theme and Activity Selections for a Cocreation Process for High Ability Students," en Trends and Advances in Information Systems and Technologies, Cham: Springer, 2018. pp. 851-858.

https://doi.org/10.1007/978-3-319-77712-2_80 
Arquitectura de servicios adaptativos para un proceso de co-creación con estudiantes con altas capacidades

[37] S. Barab y K. Squire, "Design-Based Research: Putting a Stake in the Ground," J. Learn. Sci., vol. 13, no. 1, pp. 1-14, Nov. 2004. https://doi.org/10.1207/s15327809jls1301_1

[38] F. Wang y M. J. Hannafin, "Design-based research and technology-enhanced learning environments," Educ. Technol. Res. Dev., vol. 53, no. 4, pp. 5-23, Dec. 2005.

https://doi.org/10.1007/BF02504682
[39] S. Weibelzahl, "Evaluation of Adaptive Systems," en User Modeling 2001, Berlin Heidelberg: Springer, 2001. pp. 292-294. https://doi.org/10.1007/3-540-44566-8_49 